Il existe d'autres boissons orientales analogues à celles précitées, tel est le leben égyptien, issu du lait de bufflesse. Ces boissons, dont la fabrication nécessite un lait spécial, restent localisées en leur pays d'origine.

Les laits fermentés, tout en remplissant la fonction essentielle d'aliment, jouent le rôle, non moins appréciable, de thérapeute. Il importe done de développer la consommation de ces boissons dont la consécration traditionnelle orientale constitue un sûr garant de leur haute valeur.

\title{
REVUE
}

\section{LA FABRICATION DE LA LAINE ARTIFICIELLE}

\author{
par G. GÉNIN
}

Ingénieur chimiste E. P. C.

Nous avons déjà, à plusieurs reprises, donné quelques indications sur la fabrication de la laine artificielle en Italie et sur les travaux entrepris par M. A. FerRetri pour la "Snia Viscosa",

Rappelons que c'est à la suite d'études comparatives faites entre les différentes protéines animales et végétales que cet auteur avait été conduit à constater l'analogie qui existe entre la caséine et la protéine de la laine, d'où il avait déduit qu'il devait être possible de préparer une laine artificielle en partant de caséine.

Il existe néanmoins certaines différences au point de vue de la composition entre la laine et la caséine. La laine est une scléroprotéine, tandis que la caséine est une phosphoprotéine. Il existe également d'autres différences au point de vue de la teneur en acides aminés et, d'après Fischer, le groupe acide dans la caséine prédomine, alors que c'est l'inverse dans la laine.

La laine artificielle n'a pas encore fait l'objet d'importants travaux au point de vue chimique, mais on peut penser que plus cette industrie nouvelle élargira ses bases, plus les progrès qu'on pourra lui apporter permettront de rapprocher la laine artificielle préparée à base de caséine de la laine naturelle.

Il a été jusqu'ici publié bien peu de renseignements concernant la fabrication de la fibre Lanital, nom que l'on a donné à cette laine artificielle, en particulier les aspects technologiques et chimiques de cette fabrication sont encore peu connus. Le lait est tout d'abord écrémé dans des séparateurs centrifuges qui donnent un lait écrémé ne contenant pas plus de $0,001 \%$ de matière grasse. On le coagule ensuite par addition d'acide approprié et la caséine précipitée est lavée avec soin, puis comprimée pour éliminer l'excès d'humidité. 


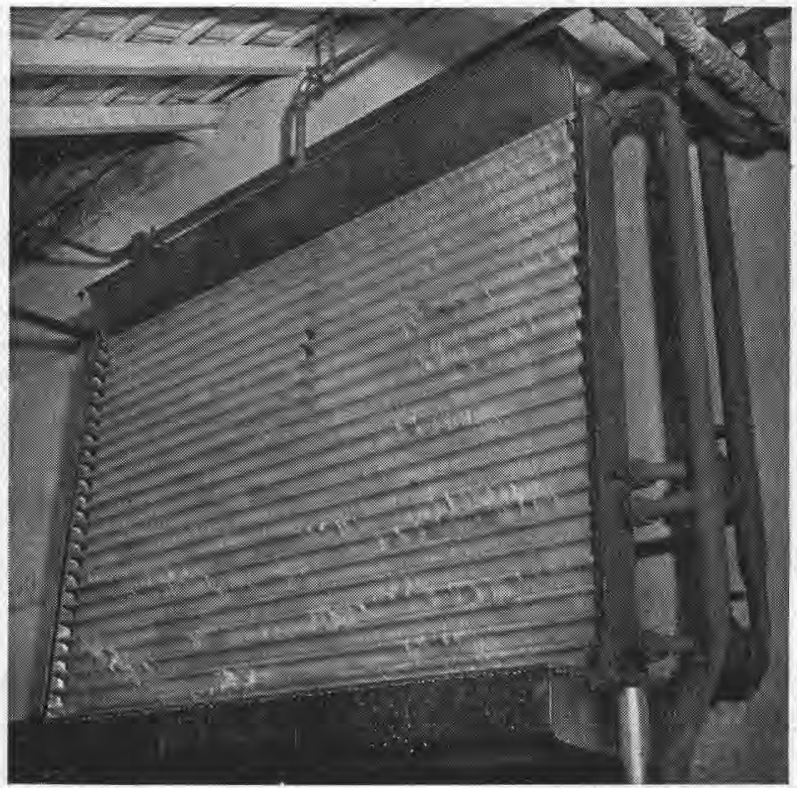

(Cliché aimablement prêté par The Industrial Chemist, London.

Fig. 1. - Réfrigérant installé à la Snia Viscosa.

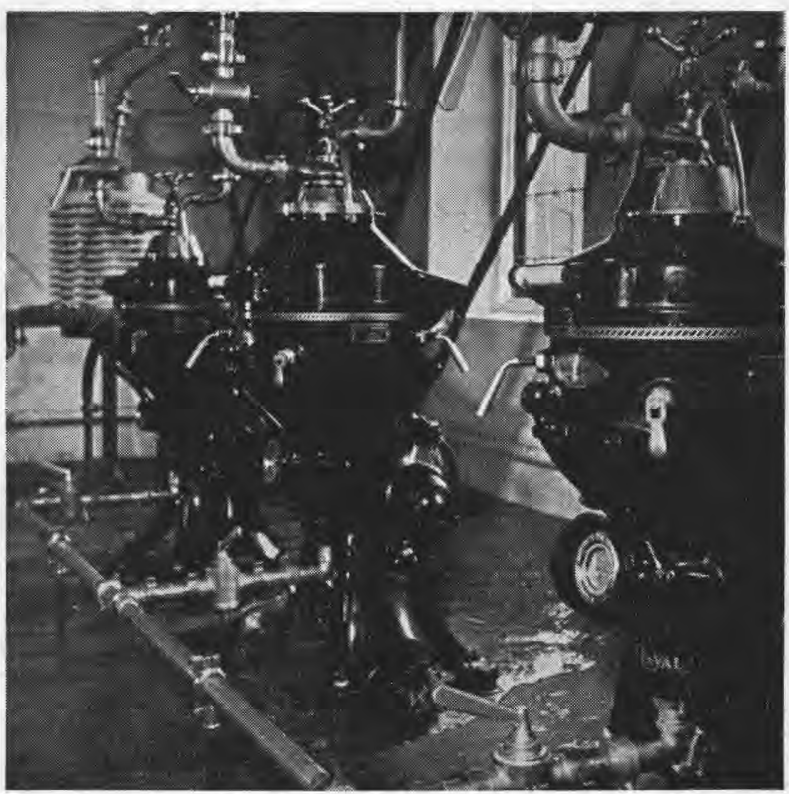

(Cliché aimablement prêté par The Industrial Chemist, London.

Fig. 2. - Groupe de centrifuges pour la séparation de la crème. 


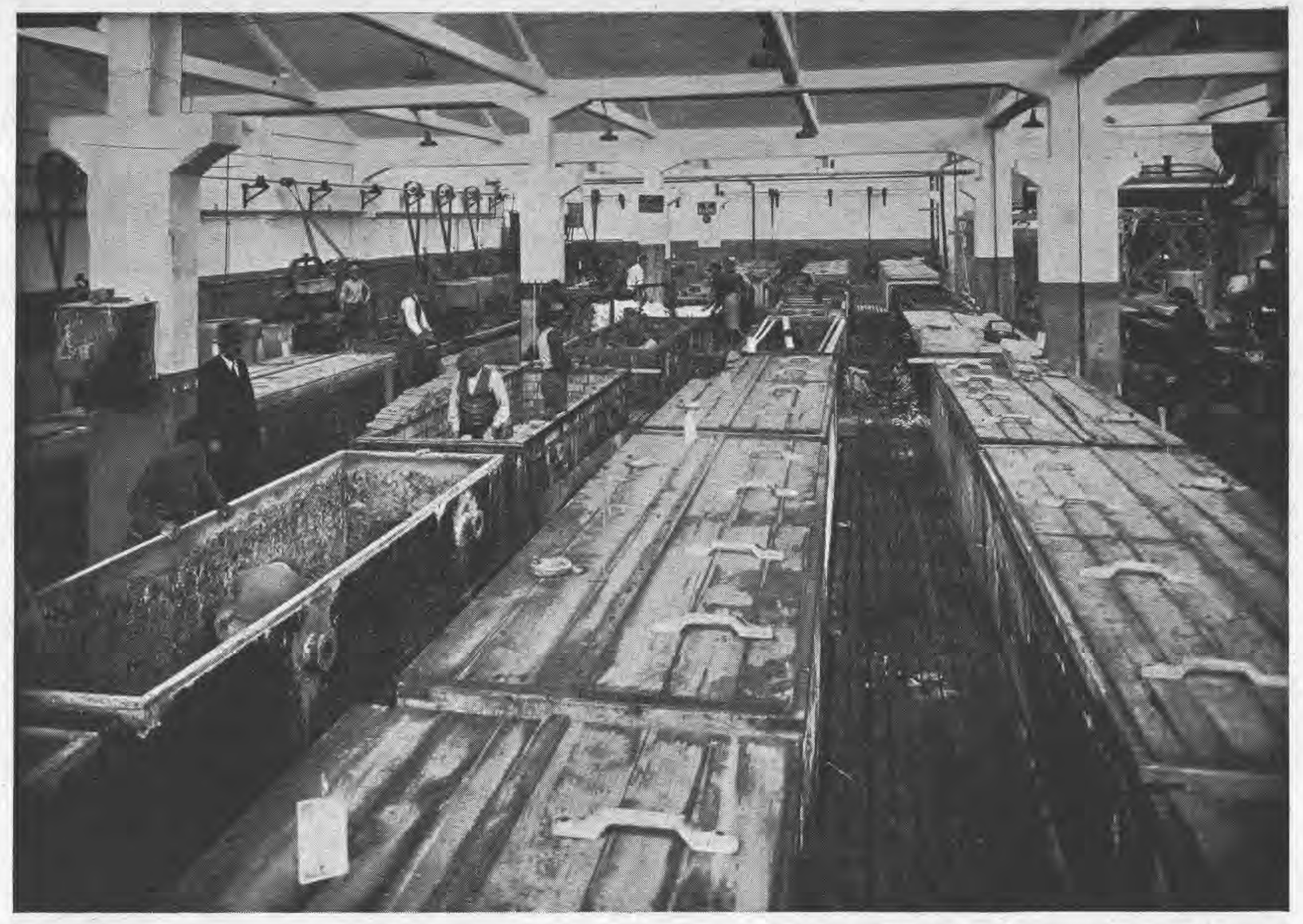

(Cliché aimablement prêté par The Industrial Chemist, London.) Fig. 3. - Batterie de cuves dans lesquelles le lait est coagulé par un acide. 


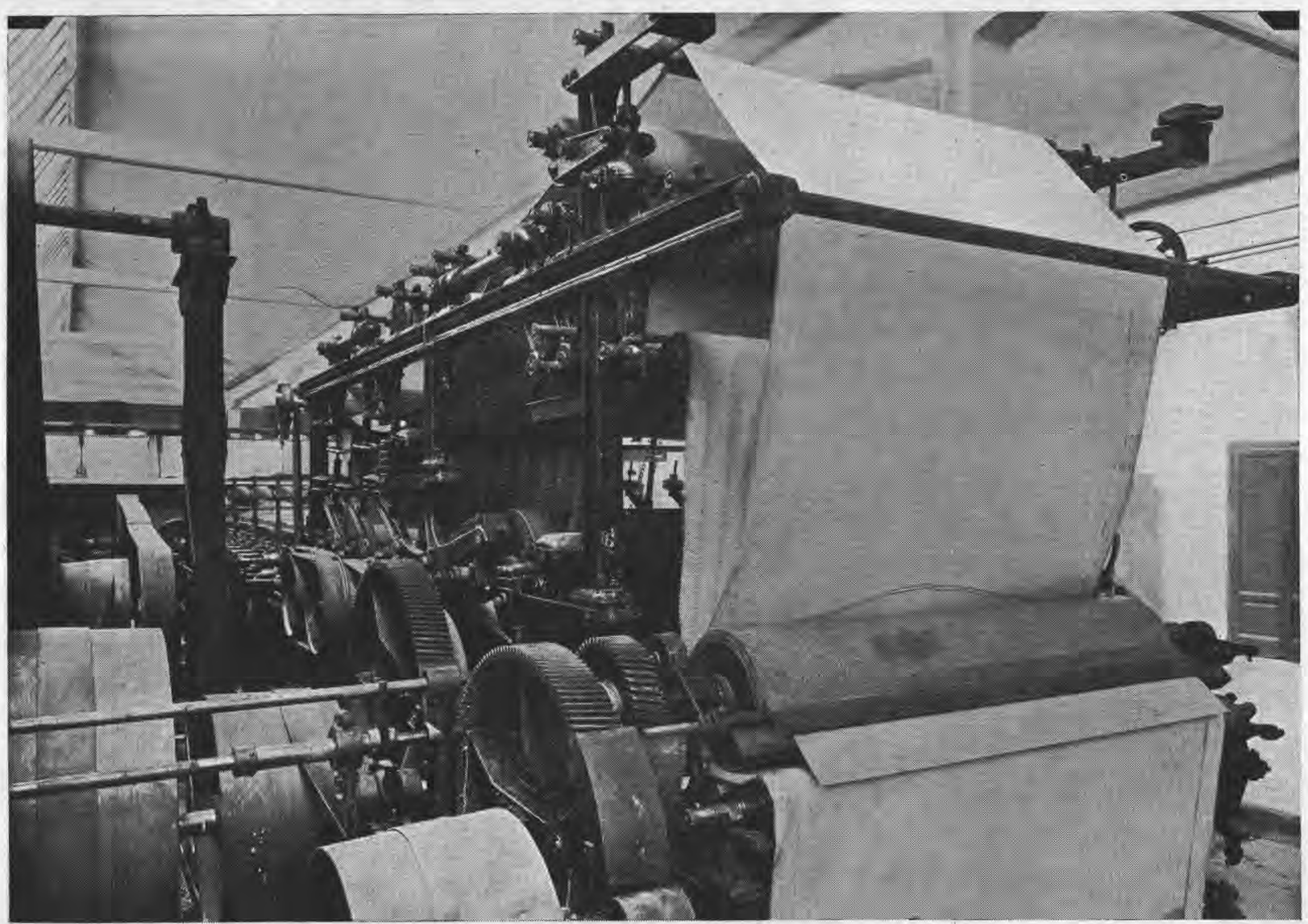

Cliché aimablement prêté par The Industrial Chemist, London.) Fig. 4. - Appareil pour le séchage de la caséine coagulée. 


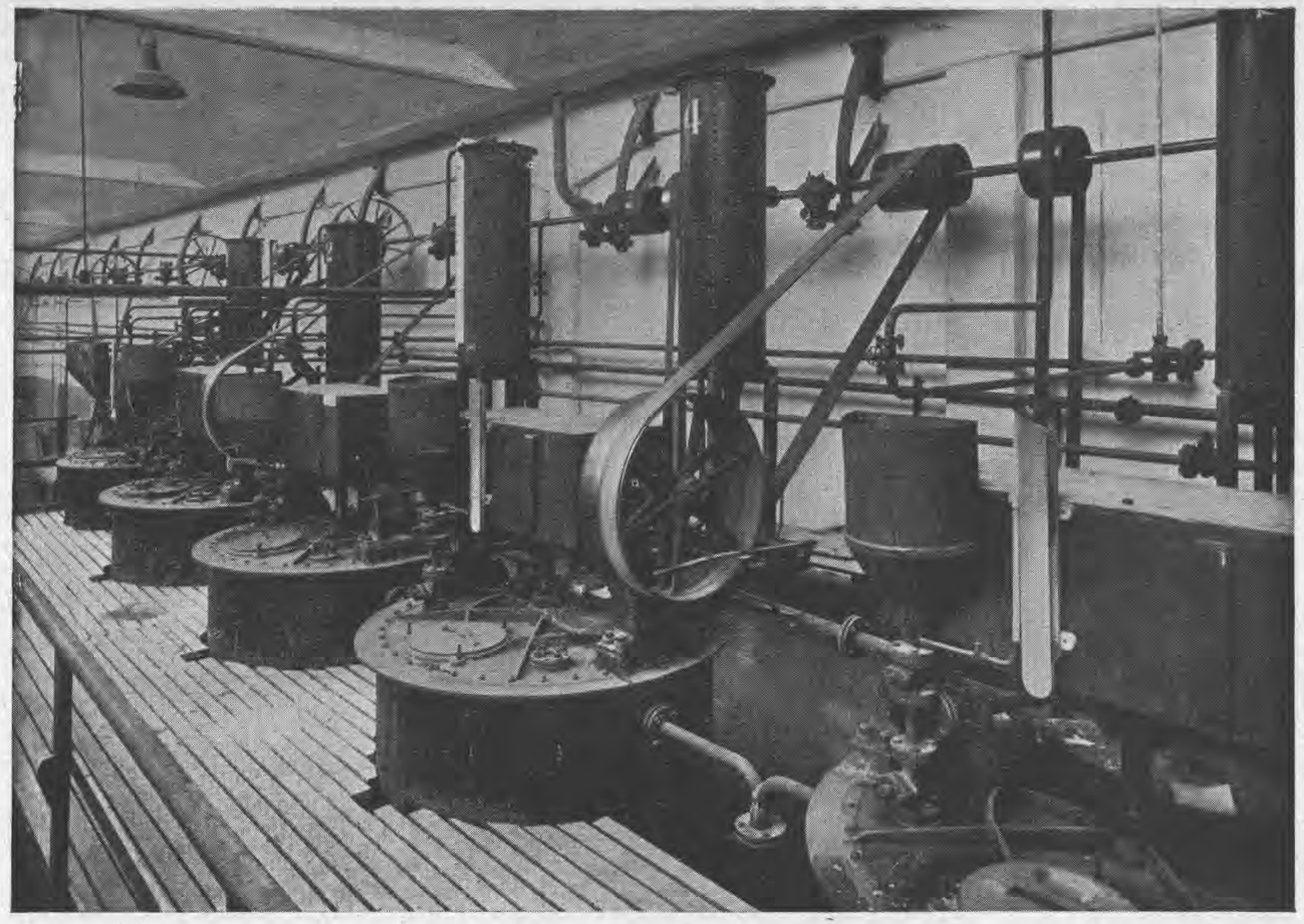

(Cliché aimablement prêté par The Industrial Chemist, London.)

Fig. 5. - Mélangeurs avec agitateurs à commande par courroie. 
La caséine encore légèrement humide est alors, soit transformée immédiatement, soit séchée en la faisant passer par l'intermédiaire des rubans transporteurs dans des chambres de séchage, parcourues.

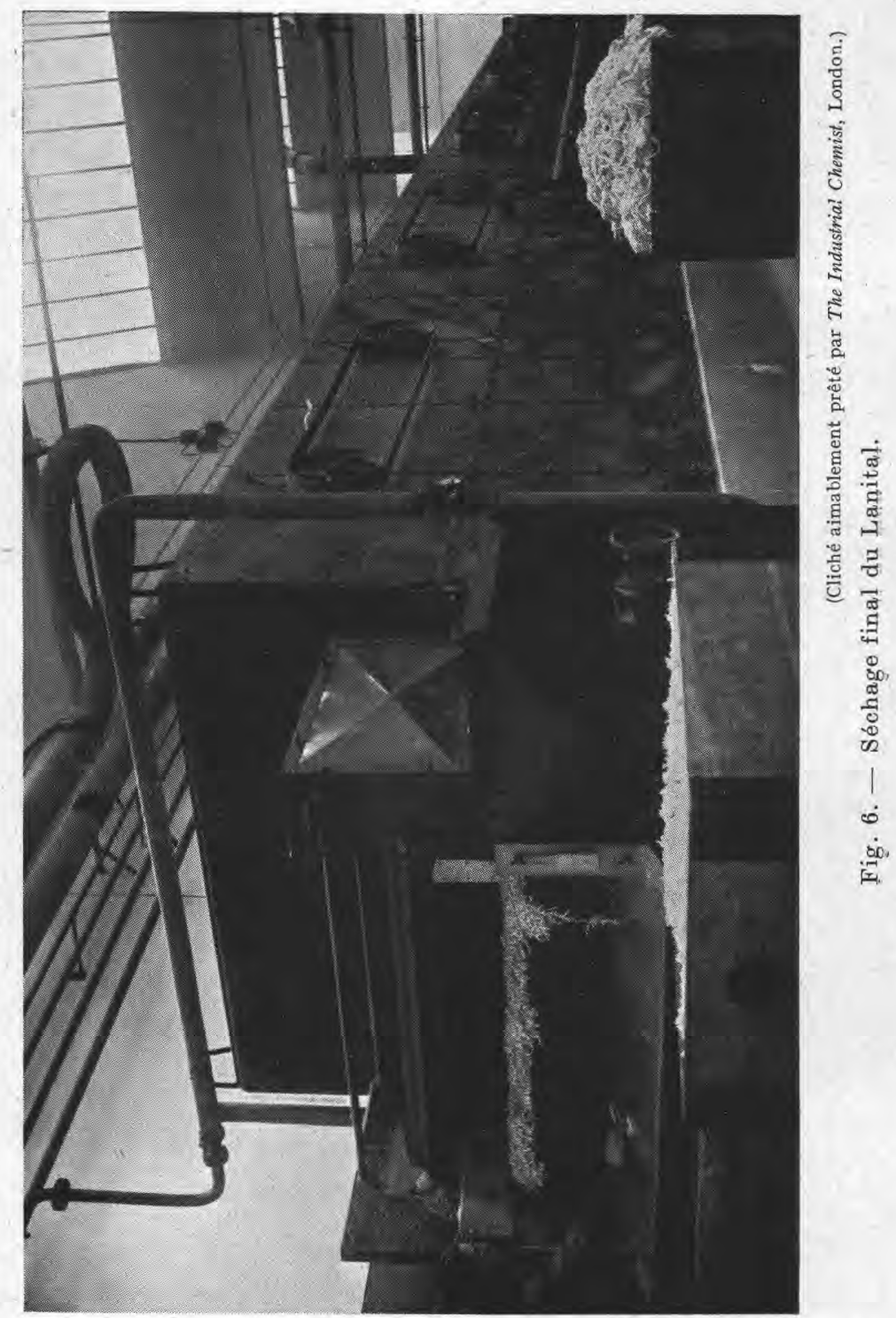

par un courant d'air chaud. Lorsque la caséine a été séchée en vue: de la stocker, il faut au moment de son emploi la réhumidifier à nouveau avant l'addition des réactifs. On prépare alors une solution. 
de caséine et le filage s'effectue suivant un procédé analogue à celui qui est utilisé dans l'industrie de la soie artificielle (soie Viscose).

On utilise à cet effet des filières dont les orifices ont des diamètres compris entre $0,02 \mathrm{~mm}$, et $0,03 \mathrm{~mm}$. Le fil est coagulé, puis découpé et lavé à de multiples reprises et enfin séché. On obtient une masse ayant l'apparence d'une laine mérinos de bonne qualité.

Voici, d'après Menozzi, l'analyse comparée du Lanital et de la laine :

\begin{tabular}{|c|c|}
\hline Carbone... & 53,00 \\
\hline Hydrogène . . . . . . . . . . . . . . . . & 7,00 \\
\hline Oxygène $\ldots \ldots \ldots \ldots \ldots \ldots$ & 23,00 \\
\hline Azote ...................... & 15,50 \\
\hline Soufre ................... & 0,70 \\
\hline
\end{tabular}

Si on soumet le Lanital à l'action de la flamme, le produit brûle lentement en laissant un résidu semblable à celui qu'on obtient en brûlant de la laine naturelle. Dans la soude caustique, le Lanital se dissout à chaud, mais moins rapidement que la laine naturelle. Après avoir fait bouillir un échantillon de Lanital dans une solution savonneuse alcaline pendant 3 heures, on a constaté que le produit avait perdu environ $1 \%$ de son poids, tandis qu'un échantillon de laine traité dans les mêmes conditions avait perdu 10 fois plus.

Au point de vue industriel, le Lanital a des propriétés très voisines de celles de la laine. Il peut être teint en particulier comme la fibre animale, mais comme la surface de la fibre est beaucoup plus unie, elle ne se feutre pas aussi facilement que la laine, ce qui dans certains cas peut présenter un avantage.

\section{BÍBLIOGRAPHE ANALYTIQLE}

\section{JOURNAUX, REVUES, SOCIÉTÉS SAVANTES ALIMENTATION DES ANIMAUX}

\section{Engels (0.). - Préparation et appréciation par des méthodes chimiques des aliments conservés en silo. Deut. landw. Tierzucht., t. XL, 1936, p. 577-579.}

L'A. a étudié en détail la préparation et la conservation en silo des aliments destinés au bétail.

Au cours de cette conservation, il doit se former des proportions importantes d'acide lactique, très peu d'acide acétique et pour ainsi dire pas d'acide butyrique. Pour doser ces acides, la méthode suivante est recommandée : on agite 100 grammes du produit finement divisé placé dans un flacon de 1 litre pendant une heure avec de l'eau distillée en utílisant de préférence un appareil automatique. On laisse ensuite reposer pendant plusieurs heures en agitant 